\title{
El Preuniversitario: un ejemplo de Educación No Formal. El caso del Preuniversitario PEDRO DE VALdivia.
}

\author{
The Pre-College Institute. Pedro de Valdivia Pre-College \\ INSTITUTE: AN EXAMPLE OF NON-FORMAL EDUCATION.
}

\author{
Alonso Morgado Alcayaga \\ Especialista en Administración Educacional \\ Preuniversitario Pedro de Valdivia \\ Alameda 155, Santiago Centro \\ amorgado@pedrodevaldivia.cl
}

Resumen: En este artículo se define el instituto preuniversitario como un ejemplo de educación no formal y se analiza el caso del Preuniversitario Pedro de Valdivia, su desenvolvimiento histórico y sus rasgos más sobresalientes, describiendo para ello el sistema de selección para las universidades chilenas y sus variaciones a lo largo del tiempo. Se señala, además, el rol que podría jugar la educación no formal destacando su función complementaria a la educación formal.

Palabras claves: educación no formal, preuniversitario, sistema de selección para la educación superior, Pruebas de Selección Universitaria, ranking de notas.

Abstract: In this article the pre-college institute is pointed out as an example of non-formal education Pedro de Valdivia Pre-College Institute is analyzed by showing its historical development and its more outstanding features. This, in relation to the system of selection for entrance to the Chilean universities and its variations in the course of time. Also, the role that non-formal education might play is signalized by its complementary function to formal education.

Keys words: non-formal education, pre-college institute, system of selection for entrance to higher education, tests for college entrance, school grades ranking.

\section{INTRODUCCIÓN}

Una de las clasificaciones más generales de los tipos de educación existentes es la que distingue tres grandes grupos:

a) Educación formal: planificada y sujeta a leyes de la República.

b) Educación no formal: planificada, no sujeta a leyes de la República.

c) Educación informal: no planificada ni sujeta a leyes de la República. 
A la educación formal pertenecen todos los establecimientos educacionales regidos por leyes u otras normas de carácter legal, entre los que podemos mencionar escuelas, colegios, liceos, centros de formación técnica, institutos profesionales y universidades. La certificación del cumplimiento de los planes educacionales por parte del alumno tiene trascendencia para su vida laboral y social.

El aparato legal que rige a este tipo de instituciones garantiza en gran medida el cumplimiento de los fines propuestos, pero administrativamente imprime un sello de gravedad que dificulta el dinamismo con que estos establecimientos deberían adaptarse a los cambios que impone una sociedad en constante desarrollo.

El caso concreto de Chile ha presentado una evolución hacia la complejidad debido a una serie de reformas educacionales durante los últimos cuarenta años, especialmente por los cambios de la educación superior, cuando fueron atomizadas las grandes universidades nacionales (U. de Chile y U. Técnica del Estado) y se permitió la creación de nuevas universidades privadas, aunque sin fines de lucro (este era el espíritu de la ley, aunque no se ha cumplido todavía). Igualmente gravitante fue la municipalización de las escuelas y liceos fiscales, perdiendo así la supervisión directa del Estado, o la creación de los establecimientos educacionales subvencionados, que tienen en sus aulas actualmente a la mayoría de los estudiantes de básica y media.

Las grandes problemáticas de la educación nacional, equidad y calidad, no se han resuelto con las medidas neoliberales ni con las reformas incoadas por distintos gobiernos a partir de 1973. Sin embargo, lo que aumentó sensiblemente fue la "cobertura", ya que la oferta educacional, sobre todo en su nivel terciario, aumentó incluso a límites no recomendables, ya que el mercado no ha crecido al mismo ritmo ni requiere las especializaciones que se están generando en la actualidad. Uno de los graves problemas de nuestro sistema es la relación entre profesionales y técnicos, ya que se observa sobreabundancia de los primeros (al menos en ciertas áreas) y escasez de los segundos.

Los requisitos de ingreso a la educación superior también han ido variando, lo que ha generado el surgimiento de un tipo de establecimiento especializado en apoyar al alumno para este fin específico, como veremos más adelante.

La educación no formal, por otra parte, constituye una oferta distinta a la educación formal, pero muchas veces en forma sustitutiva o complementaria. 
La mayor diferencia que presenta respecto a la educación formal es el hecho de no estar sometida a regulación de las leyes del país en lo que respecta al aspecto educacional propiamente tal y de administración. Esto permite la creación de sistemas de gestión más ágiles y una más rápida y efectiva adaptación a los cambios sociales y a las exigencias del mercado.

La educación que se imparte en los establecimientos no formales es planificada, pero está exenta de las exigencias legales de los establecimientos formales, que involucran también la estructura administrativa. Los procesos que se viven en establecimientos de ambas naturalezas son los mismos, pero la forma de desarrollarlos puede ser muy distinta.

Por último, la educación informal es aquella cuya enseñanza no está planificada ni responde a requisitos legales, sino que se produce en la práctica misma. Un caso conocido es un viaje, en el cual una persona puede aprender muchas cosas que no obedecen a una pauta diseñada previamente.

\section{EL SISTEMA DE SELECCIÓN DE INGRESO A LA EDUCACIÓN SUPERIOR Y LOS PREUNIVERSITARIOS.}

Señalábamos que la educación no formal puede sustituir o complementar aspectos de la educación formal. Los institutos preuniversitarios surgieron como una respuesta a la necesidad de preparar las pruebas de selección universitaria y su aparición coincide con la instauración de la Prueba de Aptitud Académica (P.A.A.) en 1967. Durante la vigencia del bachillerato los postulantes contrataban los servicios de profesores particulares. Posteriormente, cuando entró en vigencia la P.A.A. nacen los primeros preuniversitarios (Lucz - Ligeti, Indescu), cuya función ha ido variando sensiblemente a lo largo de los años.

La Prueba de Aptitud Académica (P.A.A.) tuvo una vigencia de más de treinta años, hasta ser reemplazada por las Pruebas de Selección Universitaria (P.S.U.). Este reemplazo ha causado polémicas que aún se mantienen y el período de transición fue un escándalo mediático. El primer nombre que recibió la P.S.U. fue S.I.E.S (Sistema de Ingreso a la Educación Superior)., posteriormente recibió en la prensa el nombre no oficial de P.A.T. (Prueba de Admisión de Transición) y, por último, recibió la denominación que todavía conserva.

La P.A.A. era una batería de ensayos: Aptitud Verbal, Aptitud Matemática (ambas obligatorias para postular a cualquier carrera universitaria) y las Pruebas de Conocimientos Específicos (Biología, Física, Química, Matemática, 
Ciencias Sociales y un tiempo la de Arte). La P.A.A. tenía por objetivo ser predictiva del rendimiento académico del postulante en la educación superior. Fue un sistema dinámico, que tuvo muchos cambios en su historia. Por ejemplo, la primera versión de la prueba de Aptitud Verbal tenía los siguientes tipos de ejercicio: antónimos, completación de oraciones, completación de analogías y comprensión de lectura. En su última versión sólo conservaba comprensión de lectura.

Durante un tiempo también se exigió como obligatoria la prueba de conocimientos de Historia y Geografía de Chile, a la cual se le asignaba generalmente un 10\% de la ponderación total para el puntaje de presentación a las universidades.

El año 2003 se comienza a utilizar la P.S.U., que varió su objetivo y trató, más que de predecir el rendimiento académico en la universidad, medir la calidad de la enseñanza media. Las mismas críticas que se hacían a la P.A.A. se han incrementado en la P.S.U.: discriminación de género (los hombres obtienen mejores puntajes que las mujeres), discriminación por tipo de establecimiento educacional del postulante (los mejores resultados los obtienen los alumnos de colegios particulares pagados, con una brecha importante respecto a los de establecimientos particulares subvencionados o municipales), diferencias de acuerdo al estrato socio-económico del estudiante (hay una correlación directa entre puntaje y situación económica), etc.

En los últimos años, buscando según sus defensores mayor equidad, se ha incrementado la importancia de las notas de la enseñanza media. Tradicionalmente se asignaba entre un 20\% y un 30\% al puntaje de las notas (la gran mayoría un 20\%). Actualmente se utiliza un ranking que bonifica los puntajes por notas de los alumnos que estén por sobre el promedio en su liceo o colegio, sin importar la calidad ni la tradición de ese establecimiento educacional. La ponderación de las notas y el ranking llega generalmente al $50 \%$ y, en consecuencia, las pruebas de selección sólo se ponderan en un 50\%, lo que significa una merma en su importancia que históricamente fue de un $80 \%$. Esta medida ha perjudicado sensiblemente a los alumnos de los liceos emblemáticos y a los de colegios exigentes y ha provocado reacciones como la inflación de notas, medida que adoptaron algunos liceos municipales.

La última medida del DEMRE (Departamento de Evaluación, Medición y Registro Educacional de la Universidad de Chile), señalando como causa la cautela de la privacidad de las personas, consistió en no hacer públicos los 
resultados de las P.S.U. del último año (admisión 2016), lo que ha impedido a los preuniversitarios evaluar su efectividad académica. Aparte de esto, y sin motivos explícitos, se ha impedido a las universidades dar a conocer el puntaje de corte de cada carrera, lo que perjudica a los futuros postulantes. Se había prometido hacer pública esta información en un plazo que ya venció.

Es importante señalar que Chile es el único país que relaciona el puntaje de las pruebas de admisión a las universidades con el aspecto económico. Considerando que las regalías económicas están asociadas a los puntajes más altos y considerando que está históricamente probado, año tras año, que los puntajes más altos son obtenidos por los alumnos provenientes de los estratos socio-económicos más altos, lo que se ha hecho en realidad es aumentar la inequidad del sistema desde un punto de vista social.

De lo anterior se podría inferir que el tema de la equidad ha ido por un camino sinuoso y errático que debe ser rectificado lo antes posible. La solución está, obviamente, no sólo en las pruebas de selección y el tratamiento de las notas de enseñanza media, sino mucho antes, en las condiciones socioeconómicas que permitan la igualdad de oportunidades.

\section{EL CASO DEL PREUNIVERSITARIO PEDRO DE VALDIVIA}

El Preuniversitario Pedro de Valdivia (PPV) nace el año 1978, aunque existe una fundación previa como Preuniversitario Providencia, en 1977. Ideado por dos jóvenes estudiantes de ingeniería civil de Universidad de Chile, tuvo desde sus inicios vocación por el éxito académico de sus estudiantes, estableciendo como indicadores de calidad académica los puntajes en las pruebas de selección universitaria, el ingreso de sus alumnos a la universidad y la cantidad de Puntajes Máximos Nacionales obtenidos. En este sentido, la orientación de los esfuerzos no ha sido la competencia con otros preuniversitarios, sino la superación de sus propias marcas.

Como consecuencia, las formas de publicidad empleadas han sido preferentemente de tipo testimonial. Los logros obtenidos por los alumnos se dan conocer públicamente, previa autorización, resaltando los Puntajes Máximos Nacionales (más del 50\% del total de la promoción durante más de veinte años), el ingreso a las carreras de más alta exigencia (por ejemplo, más del 50\% de aceptados en Medicina en las universidades llamadas tradicionales) y gran número de puntajes sobre 700 puntos. El preuniversitario no alcanza a tener el 6\% de los estudiantes que rinden las P.S.U., no tiene cobertura nacional 
y no tenía sedes en los sectores más altos de Santiago, donde se concentran los colegios más caros. Sólo este año se abrirán sedes en San Carlos de Apoquindo y La Dehesa.

Estos logros constituyen la gran fortaleza del PPV y logró imponerse en una industria que ha sido dinámica. Grandes preuniversitarios desaparecieron por distintas razones (CEACI, Andrés Bello, etc.), pero han surgido con fuerza otros y algunos sustitutos (profesores particulares, cursos en línea, grupos de estudio, etc.). Su cuerpo docente ha sido estable, aunque las primeras generaciones de profesores ya han comenzado a jubilarse. Más del 5\% de sus docentes tienen estudios de postgrado y la empresa impulsa y apoya el estudio individual y la capacitación permanente.

La historia del PPV sirve de ejemplo para poder apreciar cómo las instituciones no formales de educación deben adaptarse a los cambios en forma dinámica y eficiente. Si no tienen la capacidad de hacerlo, desaparecen rápidamente.

Los grandes hitos del PPV están relacionados tanto con la dinámica de desarrollo interno como son los grandes cambios que ha sufrido nuestro país en sus 38 años de existencia. Vamos a examinar separadamente estos hitos.

\section{CRECIMIENTO INTERNO}

El crecimiento interno tiene dos dimensiones: geográfico y de oferta de programas.

La primera sede del PPV estaba situada en Pedro de Valdivia con Bilbao, comuna de Providencia (hoy Sede Providencia). Su público era mayormente estudiantes provenientes de sectores pudientes y de colegios particulares pagados. El énfasis estaba en la ejercitación, en la metodología de resolución de los problemas y en el manejo del tiempo.

Al abrirse en 1980 la Sede Brasil, la incorporación de alumnos del sistema fiscal o, posteriormente, municipalizado, mostró una nueva realidad. Se hizo necesario comenzar a llenar las lagunas en los contenidos que evidenciaban los alumnos, por lo que se prestó atención a los contenidos y a las técnicas de estudio. Se creó el Departamento de Orientación, una de cuyas funciones es enseñar al alumno a estudiar. 
El año 1994 comienza la expansión por regiones. La primera sede fuera de Santiago fue la de Viña del Mar. Actualmente el PPV tiene presencia en Calama, Antofagasta, La Serena, Quillota, Quilpué, Viña del Mar, Santiago, Puente Alto, San Bernardo, Melipilla, Rancagua, Talca, Curicó, Concepción, Los Ángeles, Temuco, Valdivia, Osorno y Puerto Montt. Cada región tiene características especiales que se deben atender en forma diferenciada, lo cual ha sido un gran desafío.

Respecto a los programas de estudio, durante más de veinte años la oferta se limitaba a cursos de preparación para la P.A.A. o la P.S.U. para alumnos de cuarto medio o egresados. Hace quince años se incorporó la modalidad de cursos para tercero medio, centrados al inicio del programa en las pruebas de selección (prepararlas con más tiempo) y actualmente con foco también en subir las notas de la enseñanza media.

Debido a la importancia que asumían las notas (promedio y ranking), la misión del preuniversitario (que el alumno ingrese a la universidad) debía considerarlas también. Por tal motivo se han creado programas para primero medio y segundo medio (sólo Lenguaje y Matemática), tercero medio y cuarto medio (Lenguaje y Matemática, Biología, Física, Química y Ciencias Sociales). Los alumnos ya egresados tienen cursos en todas las asignaturas, con un programa con mayor número de horas a la semana.

También existe una oferta diferenciada para alumnos de educación técnico - profesional, para alumnos que sólo desean ejercitar (Programa Training), para alumnos que sólo desean repasar contenidos (Mentoring) y para alumnos que desean prepararse en la casa (Preuniversitario Pedro de Valdivia on line).

Existen modalidades presenciales, a distancia y mixtas. PPV cuenta con plataformas que apoyan al alumno tanto para el estudio de las asignaturas de la enseñanza media (con el fin de subir su promedio de notas), como para preparar las pruebas de selección (para subir su puntaje en ellas). El fin es el ingreso a la universidad.

Actualmente se cuenta con un modelo educativo que incorpora tecnología en aula y en línea, lo que permite al alumno acceder a cualquier hora al material tanto en contenidos como en ejercitación, evaluación y orientación. El profesor tiene también la misión de guiar a sus alumnos en el uso adecuado y pertinente de estos recursos, apoyarlo en la adquisición de técnicas de estudio y a desarrollar competencias blandas. 


\section{FACTORES EXTERNOS}

Los factores externos más importantes han sido los cambios en el sistema de ingreso a la universidad, los cambios en el sistema educacional y los cambios en la educación superior.

Elcambio deP.A.A.alaP.S.U.implicó una gran tarea, ya que las comunicaciones fueron confusas y contradictorias. Al principio se habló del S.I.E.S., que tuvo de inmediato detractores en los medios de comunicación de masas. Las preguntas que se hicieron públicas como ejemplos estaban mal construidas y en algunos casos se podían contestar sin siquiera leer el enunciado, ya que las alternativas se podían excluir entre sí. Durante algún tiempo la prensa se refirió a esta prueba como P.A.T., pero no era este su nombre oficial. Finalmente se usó el nombre P.S.U, que aún se utiliza.

Debido a la poca información sobre esta prueba, la confección de los programas de estudio era muy difícil. La forma de trabajo para hacer el material didáctico que usó el PPV consistió el atomizar los planes de estudio oficiales de la enseñanza media hasta el nivel de los conceptos. Se construyeron glosarios y con esta base se pudo anticipar en forma exitosa lo que sería finalmente la P.S.U. El modelo de pregunta se basó en las de la P.A.A. y del S.A.T. norteamericano (Scholarship Aptitude Test o Examen de Admisión Estandarizado).

Un problema adicional, de tipo comercial y publicitario, que trajo la instauración de la P.S.U. fue que la tradición de éxito del PPV se basaba en la P.A.A., pero no existía un líder de esta nueva prueba. Fue una dura lucha contra la competencia, pero los resultados obtenidos ese primer año de la P.S.U. superaban los éxitos logrados durante la vigencia de la P.A.A. Los resultados y no la publicidad garantizaron la solidez de la propuesta y al año siguiente se superó el déficit de alumnos.

El otro hito importante es de reciente data: la baja relativa de la importancia de la P.S.U. en el ingreso a la universidad y el incremento en la valoración de las notas de la enseñanza media, sobre todo mediante el ranking. Esto motivó a cambiar la misión de la empresa, añadiendo a la preparación de las pruebas de selección la potenciación del alumno como estudiante para lograr subir su promedio de notas. Por tal motivo, los programas actuales del PPV van desde primero medio hasta alumnos ya egresados.

La publicidad del PPV se ha basado tradicionalmente en datos testimoniales, como ya hemos señalado. Uno de los índices más importantes era el ingreso 
a las carreras de mayor exigencia. Cuando el porcentaje de las pruebas de selección alcanzaba un 80\%, la importancia del preuniversitario era mayor que en la actualidad, cuando llega sólo al 50\%. Como el puntaje del ranking es asignado por el promedio sin considerar la calidad del establecimiento educacional del cual procede el postulante, los alumnos de liceos y colegios más exigentes académicamente se ven perjudicados y deben subir más aún sus puntajes en las pruebas de selección. Esto generó el año 2014 el abandono en masa de alumnos de liceos emblemáticos a otros de menor exigencia. Esto actualmente ya no es posible porque se lleva un historial de cada alumno y se le asigna al puntaje considerando todos los establecimientos en los cuales ha estudiado.

La desinformación existente respecto al último proceso de selección ha impedido, como ya se señaló, la autoevaluación de los preuniversitarios, impidiendo por otra parte a los usuarios escoger documentadamente en cuál estudiar.

También los cambios en el sistema educacional han afectado de alguna manera la labor de los preuniversitarios, pero en el ámbito comercial. La existencia de establecimientos particulares pagados, particulares subvencionados y municipales, con la tendencia al aumento de los alumnos en el sistema subvencionado a costa de los liceos municipalizados, significaba que el apoderado debía ocupar recursos económicos en el copago, debido a lo cual no matriculaba a su hijo en el preuniversitario o tomaba menos cursos.

Por último, un factor que alteró gravemente el funcionamiento de los preuniversitarios fue la apertura de las universidades privadas no tradicionales. Esto significó al inicio de este sistema la disminución notoria de alumnos en los cursos de la mañana, que corresponden a los alumnos egresados. Sin embargo, esta situación se ha venido normalizando, aunque sin llegar a la cantidad de alumnos de antes, por dos motivos: en primer lugar, las universidades privadas no tradicionales han ido adquiriendo cierta valoración pública que hace de algunas de ellas establecimientos de alta demanda y puntajes de corte también altos, para lo cual el alumno requiere de la preparación de las pruebas de selección y, en segundo lugar, que debido a la importancia de las notas muchos estudiantes deciden terminar cuarto medio e ingresar al preuniversitario al año siguiente, para no perjudicar su promedio de enseñanza media. 


\section{VALORACIÓN PÚBLICA DE LOS PREUNIVERSITARIOS}

Si bien es cierto que existen muchos prejuicios respecto a los preuniversitarios (los más recurrentes de los cuales son que se aprovechan de la preparación que dan los colegios y liceos atribuyéndose los logros del alumno, que son un negocio y que son un factor de inequidad social), también es cierto que su aceptación, utilidad y valoración han venido aumentando (González, 2015) con el tiempo. Es un hecho innegable que los estudiantes logran subir sus puntajes, no sólo por adiestrarse en contestar una prueba que no se parece a los controles de la enseñanza media, sino además porque logran aprender contenidos que no habían visto en sus colegios, pese a que están en los programas oficiales. Debido a los paros de los últimos años, esto se ha hecho cada vez más frecuente.

Debido al carácter complementario de las instituciones de educación no formal y a su capacidad de adaptarse más rápidamente a los cambios que la educación formal, es difícil que desaparezcan. Por el contrario, la tendencia de estos últimos años ha sido su crecimiento, debido precisamente a las falencias de nuestros sistemas formales.

\section{CONCLUSIONES}

La educación no formal es un complemento de la educación formal. Existe una relación dialéctica entre ambas. Las críticas al sistema formal de nuestro país se ha centrado en dos grandes problemas: la falta de calidad y la falta de equidad.

La falta de calidad se suple de diversas maneras, como por ejemplo el aumento del estudio individual, la contratación de profesores particulares o el ingreso a una institución de educación no formal.

En el caso de los preuniversitarios, hay otro factor que se debe considerar en el análisis: la misión de la enseñanza media no es ni puede ser el ingreso a la universidad. No es o no debiera ser, por lo tanto, la preparación de las P.S.U. la principal preocupación de un colegio o liceo. Pero es innegable que la existencia de pruebas como las P.S.U. o el SIMCE (Sistema de Medición de la Calidad Educacional, sigla que hoy se refiere al sistema de evaluación que la Agencia de Calidad utiliza para evaluar los resultados de aprendizaje de los establecimientos educacionales de educación básica y media) alteran el funcionamiento de los establecimientos educacionales de enseñanza básica y 
media, porque los puntajes que arrojan se consideran índices de calidad, lo cual es discutible.

Aunque muchos colegios adoptan el formato de la P.S.U. en controles y ensayos, distan mucho de elaborarlos con el rigor que lo hace el DEMRE y se podría afirmar que el parecido es sólo formal (preguntas de cinco alternativas). La construcción de instrumentos como las P.S.U. requieren de una especialización que no existe en liceos y colegios.

De acuerdo a lo señalado, los alumnos de cuarto medio sienten que es necesario asistir a un preuniversitario. En el caso de los alumnos ya egresados, no tienen elección posible, porque un liceo o colegio no podría aceptarlos para satisfacer una necesidad que no está en su misión.

Respecto a la equidad, es un problema que excede largamente el ámbito educacional, ya que sus raíces se encuentran en el sistema económico que nos rige. Los resultados de las P.S.U. no hacen otra cosa que reflejar las diferencias de oportunidad de los postulantes. Como ya señaláramos, existe una correlación directa entre los puntajes obtenidos y la situación socio-económica de los postulantes (www.DEMRE.cl).

Por otra parte, las medidas adoptadas últimamente por las autoridades para mejorar la equidad mediante el ranking de notas no ataca el problema $\mathrm{y}$, a nuestro juicio, constituye una especie de manipulación que tiene efectos negativos para los alumnos de liceos y colegios más exigentes académicamente.

Se suma a esto la falta de crítica de las mismas autoridades que han mantenido un sistema de ingreso a la educación superior que asocia el puntaje a las becas y créditos. Como los alumnos de sectores socio-económicos más altos son los que obtienen los mejores puntajes, ellos mismos son los que obtienen las becas y créditos, aumentando así la inequidad. Esta situación requiere de un profundo análisis y de la voluntad política para hacer los cambios necesarios.

Al revisar la historia del Preuniversitario Pedro de Valdivia hemos podido constatar en un ejemplo concreto las características de la educación no formal y podemos concluir que ésta tiene mucho que ofrecer al sistema nacional para mejorar el estado actual. 


\section{REFERENCIAS BIBLIOGRÁFICAS}

WWW.DEMRE.cl

González, F. 2015

Valoración de los preuniversitarios en el sistema educacional chileno. Tesis para optar al grado de Doctor en Educación, Universidad Autónoma de Barcelona.

www.emol.cl 